\title{
Central Wind Power Forecasting Programs in North America by Regional Transmission Organizations and Electric Utilities
}

Subcontract Report NREL/SR-550-46763

December 2009

K. Porter and J. Rogers

Exeter Associates, Inc.

Columbia, Maryland 


\section{Central Wind Power Forecasting Programs in North America by Regional Transmission Organizations and Electric Utilities}

Subcontract Report NREL/SR-550-46763

December 2009

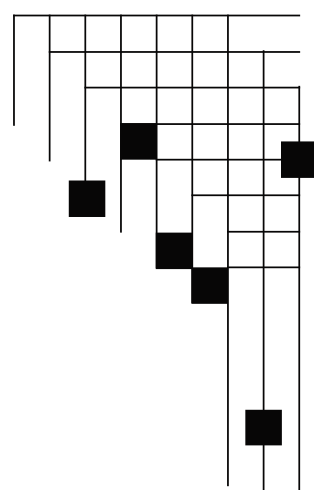

NREL Technical Monitor: J. Hein

Prepared under Subcontract No. LAM-9-99431-01

National Renewable Energy Laboratory 1617 Cole Boulevard, Golden, Colorado 80401-3393 303-275-3000 • www.nrel.gov

NREL is a national laboratory of the U.S. Department of Energy Office of Energy Efficiency and Renewable Energy

Operated by the Alliance for Sustainable Energy, LLC

Contract No. DE-AC36-08-GO28308 


\section{NOTICE}

This report was prepared as an account of work sponsored by an agency of the United States government. Neither the United States government nor any agency thereof, nor any of their employees, makes any warranty, express or implied, or assumes any legal liability or responsibility for the accuracy, completeness, or usefulness of any information, apparatus, product, or process disclosed, or represents that its use would not infringe privately owned rights. Reference herein to any specific commercial product, process, or service by trade name, trademark, manufacturer, or otherwise does not necessarily constitute or imply its endorsement, recommendation, or favoring by the United States government or any agency thereof. The views and opinions of authors expressed herein do not necessarily state or reflect those of the United States government or any agency thereof.

Available electronically at http://www.osti.gov/bridge

Available for a processing fee to U.S. Department of Energy and its contractors, in paper, from:

U.S. Department of Energy

Office of Scientific and Technical Information

P.O. Box 62

Oak Ridge, TN 37831-0062

phone: 865.576 .8401

fax: 865.576 .5728

email: mailto:reports@adonis.osti.gov

Available for sale to the public, in paper, from:

U.S. Department of Commerce

National Technical Information Service

5285 Port Royal Road

Springfield, VA 22161

phone: 800.553 .6847

fax: 703.605.6900

email: orders@ntis.fedworld.gov

online ordering: http://www.ntis.gov/ordering.htm

This publication received minimal editorial review at NREL 


\title{
Central Wind Power Forecasting Programs in North America by Regional Transmission Organizations and Electric Utilities
}

\author{
Jennifer Rogers, Exeter Associates, Inc. \\ Kevin Porter, Exeter Associates, Inc.
}

December 2009

and incorporating review comments from:

Sanjay Patil, PJM

David Edelson, New York Independent System Operator (NYISO)

David Maggio, Electricity Reliability Council of Texas (ERCOT)

Bart McManus, Bonneville Power Administration (BPA)

Mark Ahlstrom and Joe Sullivan, WindLogics

Martin Hastings and Michael Falvo, Independent Electric System Operator of Ontario

Keith Parks, Xcel Energy

Ulrich Focken, Energy \& Meteo

Alain Forcione and Jacques Bourret, Hydro Quebec

Jim Blatchford, California Independent System Operator (CAISO)

James Shen, Alberta Electric System Operator

Kenneth Pennock, Dan Meade and John Zack, AWS Truewind

Erik Ela, National Renewable Energy Laboratory (NREL)

Michael McMullen, Midwest Independent System Operator (Midwest ISO)

Barry Gilman, Southern California Edison (SCE)

Charlie Smith, Utility Wind Integration Group

The following table addresses the implementation of central wind power forecasting by electric utilities and regional transmission organizations in North America. The first part of the table focuses on electric utilities and regional transmission organizations that have central wind power forecasting in place; the second part focuses on electric utilities and regional transmission organizations that plan to adopt central wind power forecasting in 2010. Table entries are organized as follows:

- When the wind power forecast was put into operation, or will be in operation.

- What wind power forecast vendor and model is used.

- What wind power forecast tools and techniques are used.

- What applications are the wind power forecast used for.

- How wind power forecasts are paid for.

- What data is required, by wind turbine and wind project.

- How the wind power forecast is conducted.

- Whether a ramp forecast is prepared.

- How the wind power forecast was performed. 


\begin{tabular}{|c|c|c|c|}
\hline & PJM & ERCOT & Midwest ISO \\
\hline Record Peak Demand & $\begin{array}{l}144,644 \mathrm{MW} \\
\text { (August 2, 2006) }\end{array}$ & $\begin{array}{l}62,339 \mathrm{MW} \\
\text { (August 2006) }\end{array}$ & $\begin{array}{l}116,030 \mathrm{MW} \\
\text { (July } 31,2006 \text { ) }\end{array}$ \\
\hline $\begin{array}{l}\text { Available Generating } \\
\text { Capacity }\end{array}$ & $164,895 \mathrm{MW}$ & $80,076 \mathrm{MW}$ & $138,556 \mathrm{MW}$ \\
\hline Installed Wind Capacity & About $2500 \mathrm{MW}$ & $8,916 \mathrm{MW}$ & About $7200 \mathrm{MW}$ \\
\hline Area Served & $\begin{array}{l}\text { All or parts of Delaware, } \\
\text { Illinois, Indiana, Kentucky, } \\
\text { Maryland, Michigan, New } \\
\text { Jersey, North Carolina, } \\
\text { Ohio, Pennsylvania, } \\
\text { Tennessee, Virginia, West } \\
\text { Virginia and the District of } \\
\text { Columbia. }\end{array}$ & $\begin{array}{l}85 \% \text { of load in Texas; } 75 \% \\
\text { of Texas by geography. }\end{array}$ & $\begin{array}{l}\text { All or most of North } \\
\text { Dakota, South Dakota, } \\
\text { Nebraska, Minnesota, } \\
\text { lowa, Wisconsin, Illinois, } \\
\text { Indiana, Michigan and } \\
\text { parts of Montana, } \\
\text { Missouri, Kentucky, and } \\
\text { Ohio. }\end{array}$ \\
\hline $\begin{array}{l}\text { Date of Operation of } \\
\text { Wind Forecast }\end{array}$ & April 2009 & July 2008 & June 2008 \\
\hline $\begin{array}{l}\text { Wind Power Forecast } \\
\text { Vendor }\end{array}$ & Energy \& Meteo GmbH & AWS Truewind & Energy \& Meteo GmbH \\
\hline Forecast Model & Previento & eWind & Previento \\
\hline $\begin{array}{l}\text { Forecast } \\
\text { Tools/Techniques }\end{array}$ & $\begin{array}{l}\text { Physical model that uses } \\
\text { Numerical Weather } \\
\text { Prediction (NWP) } \\
\text { forecasts as input. } \\
\text { Energy \& Meteo uses } \\
\text { NWP input, a combination } \\
\text { of several numerical } \\
\text { weather models weighted } \\
\text { according to the weather } \\
\text { situation, site-specific } \\
\text { power curves based on } \\
\text { historical data, and a } \\
\text { shorter-term model (0-10 } \\
\text { hours) based on wind } \\
\text { power measurements and } \\
\text { NWPs. } \\
\text { Wind turbine deration } \\
\text { data is integrated in the } \\
\text { forecast. }\end{array}$ & $\begin{array}{l}\text { AWS Truewind uses a } \\
\text { composite of the } \\
\text { individual members of an } \\
\text { ensemble of forecasts for } \\
\text { each wind project in the } \\
\text { ERCOT territory. } \\
\text { Uses three NWP models, } \\
\text { one run every } 3 \text { hrs and } \\
\text { two run every } 6 \text { hrs; Wind } \\
\text { Generation Resource } \\
\text { (WGR) output model } \\
\text { mixed approach: some } \\
\text { statistical using WGR data } \\
\text { and some specified power } \\
\text { curve } \\
\text { Plan to use: } 9 \text { NWP model } \\
\text { ensemble run every } 6 \text { hrs; } \\
\text { single NWP model run } \\
\text { every hr (for Residual Unit } \\
\text { Commitment); Statistical } \\
\text { optimized ensemble } \\
\text { procedure that will weight } \\
\text { ensemble members } \\
\text { according to performance } \\
\text { in a rolling training } \\
\text { sample; Statistical power } \\
\text { output model: all WGRs } \\
\text { with adequate data }\end{array}$ & $\begin{array}{l}\text { Physical model that uses } \\
\text { Numerical Weather } \\
\text { Prediction forecasts as } \\
\text { input. Energy \& Meteo } \\
\text { uses NWP input, a } \\
\text { combination of several } \\
\text { numerical weather models } \\
\text { weighted according to the } \\
\text { weather situation, site- } \\
\text { specific power curves } \\
\text { based on historical data, } \\
\text { and a shorter-term model } \\
\text { (0-10 hours) based on } \\
\text { wind power } \\
\text { measurements and NWP } \\
\text { input. }\end{array}$ \\
\hline
\end{tabular}




\begin{tabular}{|c|c|c|c|}
\hline & PJM & ERCOT & Midwest ISO \\
\hline Forecast Applications & $\begin{array}{l}\text { Developing process to } \\
\text { automate the integration } \\
\text { of day-ahead wind power } \\
\text { forecast within existing } \\
\text { PJM systems to determine } \\
\text { whether there is sufficient } \\
\text { generation scheduled } \\
\text { within PJM to meet } \\
\text { expected load, transaction } \\
\text { schedules, and reserve } \\
\text { requirements. }\end{array}$ & $\begin{array}{l}\text { Day-ahead Unit } \\
\text { Commitment uses } 80 \% \\
\text { exceedance forecast for } \\
\text { day-ahead planning (i.e., } \\
80 \% \text { chance production } \\
\text { meets or exceeds wind } \\
\text { power forecast). }\end{array}$ & $\begin{array}{l}\text { Transmission security } \\
\text { planning and outage } \\
\text { coordination; and forward } \\
\text { and intra-day reliability } \\
\text { analysis. Also can use } \\
\text { wind power forecast to } \\
\text { determine impact of wind } \\
\text { variability on transmission } \\
\text { flowgates. }\end{array}$ \\
\hline $\begin{array}{l}\text { Forecast Payment } \\
\text { Method }\end{array}$ & $\begin{array}{l}\text { PJM pays for the central } \\
\text { wind power forecasting } \\
\text { service. }\end{array}$ & $\begin{array}{l}\text { ERCOT pays for the central } \\
\text { wind power forecasting } \\
\text { service. }\end{array}$ & $\begin{array}{l}\text { Midwest ISO pays for the } \\
\text { central wind power } \\
\text { forecasting service. }\end{array}$ \\
\hline $\begin{array}{l}\text { Wind Turbine Data } \\
\text { Requirements }\end{array}$ & $\begin{array}{l}\text { General turbine } \\
\text { information (class of } \\
\text { turbine, turbine capacity); } \\
\text { min/max wind speed; } \\
\text { manufacturer power } \\
\text { curves; geographic } \\
\text { location; hub height; } \\
\text { ambient temperature } \\
\text { operating limits and } \\
\text { information on installation } \\
\text { of cold weather packages. } \\
\text { Along with these turbine- } \\
\text { specific requirements, the } \\
\text { initial project data } \\
\text { required includes } \\
\text { aggregate historic power } \\
\text { output, meteorological } \\
\text { and outage data; and the } \\
\text { aggregate reactive } \\
\text { capability curve. }\end{array}$ & $\begin{array}{l}\text { The number, MW rating, } \\
\text { and model of turbines. } \\
\text { Also requires average hub } \\
\text { height of the project and } \\
\text { geographic location of the } \\
\text { center of the wind project. } \\
\text { Turbine } \\
\text { Outage/Availability data is } \\
\text { currently voluntary; } \\
\text { however ERCOT is working } \\
\text { on making it a } \\
\text { requirement. }\end{array}$ & $\begin{array}{l}\text { Not applicable - wind } \\
\text { projects do not provide } \\
\text { any turbine-specific } \\
\text { information to Energy \& } \\
\text { Meteo. } \\
\text { Wind turbine outages not } \\
\text { currently factored into the } \\
\text { wind power forecast, but } \\
\text { the Midwest ISO } \\
\text { anticipates doing so in the } \\
\text { future. }\end{array}$ \\
\hline $\begin{array}{l}\text { Wind Project Data } \\
\text { Requirements }\end{array}$ & $\begin{array}{l}\text { Real time aggregate wind } \\
\text { project MW output. } \\
\text { Must install at least one } \\
\text { meteorological tower (or } \\
\text { wind speed and direction } \\
\text { from selected wind } \\
\text { turbine anemometers and } \\
\text { wind vanes) with wind } \\
\text { speed and wind direction } \\
\text { data required; } \\
\text { temperature and pressure } \\
\text { data preferred; and } \\
\text { humidity data accepted. } \\
\text { Outage of turbines } \geq 1 \mathrm{MW} \\
\text { or an outage lasting } \\
1 \text { hour or more must be } \\
\text { reported. }\end{array}$ & $\begin{array}{l}\text { AWS Truewind Data } \\
\text { Requirements: Observed } \\
\text { Generation; Observed } \\
\text { Availability; Observed } \\
\text { Basepoint; Observed } \\
\text { Meteorological Data } \\
\text { (Wind Speed, Wind } \\
\text { Direction, Temperature, } \\
\text { and Atmospheric } \\
\text { Pressure). }\end{array}$ & $\begin{array}{l}\text { Market participants } \\
\text { required to provide non- } \\
\text { binding day-ahead } \\
\text { intermittent resource } \\
\text { forecast to Midwest ISO. } \\
\text { Midwest ISO provides the } \\
\text { latitude and longitude } \\
\text { values of each wind } \\
\text { project, the hub heights, } \\
\text { maximum and historical } \\
\text { MW output, and real-time } \\
\text { output for each wind } \\
\text { project to Energy \& } \\
\text { Meteo. Energy \& Meteo } \\
\text { then uses this information } \\
\text { to create wind power } \\
\text { forecasts. }\end{array}$ \\
\hline
\end{tabular}




\begin{tabular}{|c|c|c|c|}
\hline & PJM & ERCOT & Midwest ISO \\
\hline Description of Forecast & $\begin{array}{l}\text { Long Term: Provided } \\
\text { hourly, from } 48 \text { hours } \\
\text { ahead to } 168 \text { hours ahead. } \\
\text { Medium Term: Updated } \\
\text { from } 6 \text { hours ahead to } 48 \\
\text { hours ahead. } \\
\text { Short Term: Updated with } \\
\text { frequency of every } 10 \\
\text { min, forecast interval of } 5 \\
\text { min for next } 6 \text { hours. } \\
\text { Confidence interval } \\
\text { defined by PJM. } \\
\text { Forecast on } 5 \text { aggregation } \\
\text { levels. } \\
\text { Statistical power curve for } \\
\text { each wind project. }\end{array}$ & $\begin{array}{l}\text { Wind power forecasts are } \\
\text { updated hourly and cover } \\
1 \text { - } 48 \text { hours ahead. } \\
\text { Forecast delivered by } 15 \\
\text { minutes past each hour. } \\
\text { Though not currently } \\
\text { required, the following } \\
\text { will be a requirement of } \\
\text { the nodal market when it } \\
\text { is launched in late 2010: } \\
\text { Long-Term (one year) } \\
\text { forecasts: The long-term } \\
\text { forecast is a "typical" } \\
\text { energy profile for each } \\
\text { day of a future month out } \\
\text { to } 36 \text { months. Wind } \\
\text { developers will be } \\
\text { required to submit the } \\
\text { long-term wind power } \\
\text { forecast each month for a } \\
\text { rolling } 36 \text { month period. }\end{array}$ & $\begin{array}{l}\text { Receives hourly updated } \\
\text { forecasts from Energy \& } \\
\text { Meteo for each hour of } \\
\text { the next seven days, for } \\
\text { over } 100 \text { Commercial } \\
\text { Pricing (CP) nodes. } \\
\text { Forecasts available more } \\
\text { frequently, but Midwest } \\
\text { ISO has not yet } \\
\text { determined that need. } \\
\text { A short term forecast is } \\
\text { provided for the next } 6 \\
\text { hours and the remainder } \\
\text { of the time is considered a } \\
\text { medium/long-term } \\
\text { forecast. } \\
\text { Energy \& Meteo provides } \\
\text { forecasts at } 4 \text { levels: CP } \\
\text { nodes, zones, regions and } \\
\text { all of Midwest ISO. } \\
\text { Generally, the CP nodes } \\
\text { represent an individual } \\
\text { wind project. Three } \\
\text { different NWP models are } \\
\text { used for each of these } \\
\text { levels. Energy \& Meteo } \\
\text { also forecasts wind power } \\
\text { output as an optimal } \\
\text { combination of all three } \\
\text { forecasts. } \\
\text { Statistical power curve for } \\
\text { each wind project. }\end{array}$ \\
\hline Ramp Forecast & $\begin{array}{l}\text { Updated every } 10 \text { minutes } \\
\text { at } 5 \text { min intervals for next } \\
6 \text { hours. }\end{array}$ & $\begin{array}{l}\text { In process of developing a } \\
\text { ramp forecast. }\end{array}$ & $\begin{array}{l}\text { Several wind power } \\
\text { forecasts indicating } \\
\text { possible ramps are } \\
\text { provided; however, ramp } \\
\text { forecasting metrics are } \\
\text { still being developed. }\end{array}$ \\
\hline
\end{tabular}




\begin{tabular}{|c|c|c|c|}
\hline & PJM & ERCOT & Midwest ISO \\
\hline $\begin{array}{l}\text { Forecast Performance } \\
\text { Metrics }\end{array}$ & $\begin{array}{l}\text { For all of PJM monthly } \\
\text { averaged mean absolute } \\
\text { error (MAE) for the May } \\
2009 \text { - July } 2009 \text { period } \\
\text { ranged from } 4.9 \% \text { to } 5.1 \% \\
\text { for intra-day; } 5.9 \% \text { to } 7.9 \% \\
\text { for day-ahead; and } 5.2 \% \\
\text { to } 5.6 \% \text { for the evening } \\
\text { forecast at } 4: 00 \text { PM, which } \\
\text { covers } 8 \text { to } 32 \text { hours } \\
\text { ahead. } \\
\\
\text { The monthly averaged } \\
\text { Root Mean Squared Error } \\
\text { (RMSE) for the May } 2009 \\
\text { - July } 2009 \text { period ranged } \\
\text { from } 6.5 \% \text { to } 7.3 \% \text { for } \\
\text { intra-day; } 8.3 \% \text { to } 10.3 \% \\
\text { for day-ahead; and } 6.9 \% \\
\text { to } 7.6 \% \text { for the evening } \\
\text { forecast. Forecast } \\
\text { performance achieved } \\
\text { without meteorological } \\
\text { data. }\end{array}$ & $\begin{array}{l}\text { For all of ERCOT, monthly } \\
\text { averaged MAE for the } \\
\text { 4:30 PM system-wide day- } \\
\text { ahead forecast, for May } \\
2009-\text {-August } 2009, \text { ranged } \\
\text { from } 8.28 \% \text { to } 10.73 \% \text { of } \\
\text { capacity for all hours. }\end{array}$ & $\begin{array}{l}\text { For all of the Midwest ISO, } \\
\text { monthly averaged MAE } \\
\text { for the 4:30 PM forecast } \\
\text { for day-ahead, for May } \\
2009 \text { - July 2009, ranged } \\
\text { from } 3.3 \% \text { to } 4.5 \% \text { for all } \\
\text { hours. } \\
\text { The monthly averaged } \\
\text { RMSE for intraday ( } 0-24 \\
\text { hour) ranges between } 4 \% \\
\text { and } 7 \% \text { for the period } \\
\text { from August } 2008 \text { to } \\
\text { August } 2009 \text {. } \\
\text { For monthly RMSE for } \\
\text { day-ahead ( } 24-48 \text { ) } \\
\text { ranges from } 5 \text { to } 10 \% \text {. This } \\
\text { is the accuracy for the first } \\
\text { year after the setup. }\end{array}$ \\
\hline
\end{tabular}




\begin{tabular}{|c|c|c|c|c|}
\hline & NYISO & CAISO & SCE & Hydro-Québec \\
\hline $\begin{array}{l}\text { Record Peak } \\
\text { Demand }\end{array}$ & $\begin{array}{l}\text { 33,939 MW } \\
\text { (August 2, 2006) }\end{array}$ & $\begin{array}{l}\text { 50,270 MW } \\
\text { (July 24, 2006) }\end{array}$ & $\begin{array}{l}\text { 23,303 MW } \\
\text { (August 31, 2007) }\end{array}$ & $\begin{array}{l}37,230 \mathrm{MW} \\
\text { (January 16, 2009) }\end{array}$ \\
\hline $\begin{array}{l}\text { Available } \\
\text { Generating } \\
\text { Capacity }\end{array}$ & $\begin{array}{l}38,190 \mathrm{MW} \text { (Summer } \\
2009)\end{array}$ & $\begin{array}{l}48,954 \mathrm{MW} \text { (does } \\
\text { not include } 10,350 \\
\text { MW of net imports). }\end{array}$ & $\begin{array}{l}\text { SCE considers data } \\
\text { as confidential. }\end{array}$ & $43,664 \mathrm{MW}$ \\
\hline $\begin{array}{l}\text { Installed Wind } \\
\text { Capacity }\end{array}$ & $1,275 \mathrm{MW}$ & $\begin{array}{l}\text { 2,953 MW (CAISO } \\
\text { total); } 1,005 \mathrm{MW} \text { in } \\
\text { CAISO's wind } \\
\text { forecasting program. }\end{array}$ & $1,073 \mathrm{MW}$ & $657 \mathrm{MW}$ \\
\hline Areas Served & New York & $\begin{array}{l}75 \% \text { of California } \\
\text { load }\end{array}$ & $\begin{array}{l}50,000 \text { square mile } \\
\text { area of California, } \\
\text { excluding the City } \\
\text { of Los Angeles and } \\
\text { certain other cities. }\end{array}$ & Quebec, Canada \\
\hline $\begin{array}{l}\text { Date of Operation } \\
\text { of Wind Forecast }\end{array}$ & $\begin{array}{l}\text { June } 2008 \text { - for } \\
\text { dispatch decisions; } \\
\text { May } 2009 \text { - for } \\
\text { individual wind plant } \\
\text { economic dispatch } \\
\text { decisions }\end{array}$ & June 2004 & November 2000 & November 2006 \\
\hline $\begin{array}{l}\text { Wind Power } \\
\text { Forecast Vendor }\end{array}$ & AWS Truewind & AWS Truewind & AWS Truewind & $\begin{array}{l}\text { Environment Canada: } \\
\text { operational GEM } \\
\text { 15-km NWP }\end{array}$ \\
\hline Forecast Model & eWind & eWind & eWind & $\begin{array}{l}\text { Anemos/WPPT; HQ } \\
\text { proprietary models; } \\
\text { Forecasting tools } \\
\text { operated by HQ. }\end{array}$ \\
\hline $\begin{array}{l}\text { Forecast } \\
\text { Tools/Techniques }\end{array}$ & $\begin{array}{l}\text { Uses ensemble } \\
\text { forecasts and } \\
\text { statistical analysis to } \\
\text { prepare wind power } \\
\text { forecast. Uses the } \\
\text { following inputs: grid } \\
\text { point output from } \\
\text { regional-scale and } \\
\text { global-scale NWP } \\
\text { models; } \\
\text { measurement data } \\
\text { from several } \\
\text { meteorological } \\
\text { sensors; high- } \\
\text { resolution } \\
\text { geographical data; } \\
\text { and meteorological } \\
\text { and generation data } \\
\text { from wind projects. }\end{array}$ & $\begin{array}{l}\text { Uses ensemble } \\
\text { forecasts and } \\
\text { statistical analysis to } \\
\text { prepare wind power } \\
\text { forecast. Uses the } \\
\text { following inputs: grid } \\
\text { point output from } \\
\text { regional-scale and } \\
\text { global-scale NWP } \\
\text { models; } \\
\text { measurement data } \\
\text { from several } \\
\text { meteorological } \\
\text { sensors; high- } \\
\text { resolution } \\
\text { geographical data; } \\
\text { and meteorological } \\
\text { and generation data } \\
\text { from wind projects. }\end{array}$ & $\begin{array}{l}\text { Uses ensemble } \\
\text { forecasts and } \\
\text { statistical analysis } \\
\text { to prepare wind } \\
\text { power forecast. } \\
\text { Uses the following } \\
\text { inputs: grid point } \\
\text { output from } \\
\text { regional-scale and } \\
\text { global-scale NWP } \\
\text { models; } \\
\text { measurement data } \\
\text { from several } \\
\text { meteorological } \\
\text { sensors; high- } \\
\text { resolution } \\
\text { geographical data; } \\
\text { and meteorological } \\
\text { and generation data } \\
\text { from wind projects. } \\
\text { SCE inputs outage } \\
\text { and curtailment } \\
\text { data both prior and } \\
\text { post-production. }\end{array}$ & $\begin{array}{l}\text { Statistical models } \\
\text { using NWP and actual } \\
\text { wind project } \\
\text { generation and } \\
\text { turbine availability as } \\
\text { inputs. Extensive R\&D } \\
\text { program on additional } \\
\text { and complementary } \\
\text { forecasting tools at } \\
\text { the Institut de } \\
\text { recherche d'Hydro- } \\
\text { Québec (IREQ), Hydro- } \\
\text { Québec's research } \\
\text { institute. }\end{array}$ \\
\hline
\end{tabular}




\begin{tabular}{|c|c|c|c|c|}
\hline & NYISO & CAISO & SCE & Hydro-Québec \\
\hline $\begin{array}{l}\text { Forecast } \\
\text { Applications }\end{array}$ & $\begin{array}{l}\text { Used to review day- } \\
\text { ahead unit } \\
\text { commitment } \\
\text { schedules to ensure } \\
\text { enough generation is } \\
\text { committed to meet } \\
\text { load forecast. Also } \\
\text { used in real time to } \\
\text { make real-time } \\
\text { commitment and } \\
\text { dispatch decisions. }\end{array}$ & $\begin{array}{l}\text { Used in hour-ahead } \\
\text { market, as } \\
\text { Participating } \\
\text { Intermittent } \\
\text { Resource Program } \\
\text { (PIRP) participants } \\
\text { must bid wind power } \\
\text { forecast. Day-ahead } \\
\text { wind power forecast } \\
\text { advisory. }\end{array}$ & Energy scheduling. & $\begin{array}{l}\text { Day-ahead scheduling; } \\
\text { Intra-day } \\
\text { rescheduling; short- } \\
\text { term maintenance } \\
\text { scheduling. The wind } \\
\text { power forecast is } \\
\text { taken into account in } \\
\text { the intra-day } \\
\text { scheduling (generation } \\
\text { scheduled to meet } \\
\text { expected load, } \\
\text { transaction schedules, } \\
\text { and reserve } \\
\text { requirements). }\end{array}$ \\
\hline $\begin{array}{l}\text { Forecast Payment } \\
\text { Method }\end{array}$ & $\begin{array}{l}\text { Fee assessed to each } \\
\text { wind project. Charge } \\
\text { includes the sum of a } \\
\text { monthly fee of } \$ 500 \\
\text { and a separate } \\
\text { monthly fee of } \$ 7.50 \\
\text { per MW of } \\
\text { nameplate capacity. } \\
\text { Fees are subject to } \\
\text { change as more wind } \\
\text { projects are added. }\end{array}$ & $\begin{array}{l}\text { Fee assessed on PIRP } \\
\text { participating } \\
\text { intermittent } \\
\text { resources of } \\
\text { \$0.10/MWh, and the } \\
\text { CAISO covers about } \\
\text { \$0.09/MWh from } \\
\text { within its operating } \\
\text { budget. } \\
\text { Proposed change: } \\
\text { Assess \$0.10/MWh } \\
\text { fee to non-PIRP } \\
\text { intermittent } \\
\text { resources as well. }\end{array}$ & $\begin{array}{l}\text { SCE pays for the } \\
\text { wind power } \\
\text { forecasting service. }\end{array}$ & $\begin{array}{l}\text { HQ pays for the wind } \\
\text { power forecasting } \\
\text { service. }\end{array}$ \\
\hline $\begin{array}{l}\text { Wind Turbine } \\
\text { Data } \\
\text { Requirements }\end{array}$ & $\begin{array}{l}\text { Turbine } \\
\text { manufacturer } \\
\text { specifications; plant } \\
\text { configuration } \\
\text { (latitude/longitude of } \\
\text { each turbine); } \\
\text { manufacturer's } \\
\text { power curve; } \\
\text { plant/turbine } \\
\text { availability; plant } \\
\text { level or turbine level } \\
\text { power output data. }\end{array}$ & $\begin{array}{l}\text { Real-time telemetry } \\
\text { data requirements: } \\
\text { wind speed, wind } \\
\text { direction, barometric } \\
\text { pressure, ambient } \\
\text { temperature, real- } \\
\text { time MW production, } \\
\text { MW production } \\
\text { revenue meter. } \\
\text { Data requirements: } \\
\text { latitude/longitude and } \\
\text { elevation of the } \\
\text { turbine hub height } \\
\text { that will be used as a } \\
\text { designated turbine. A } \\
\text { designated turbine is } \\
\text { the turbine designated } \\
\text { to send in } \\
\text { anemometry data to } \\
\text { represent a } \\
\text { surrounding group of } \\
\text { turbines. }\end{array}$ & $\begin{array}{l}\text { MW production } \\
\text { metering }\end{array}$ & $\begin{array}{l}\text { Operational planning } \\
\text { data (planned turbine } \\
\text { availability); turbine } \\
\text { specifications (power } \\
\text { curve, control system, } \\
\text { cold weather } \\
\text { packages, etc); real- } \\
\text { time data including } \\
\text { kW of active power, } \\
\text { nacelle direction, } \\
\text { blade position, } \\
\text { temperature at nacelle } \\
\text { level, wind speed, } \\
\text { wind direction, and } \\
\text { turbine status. }\end{array}$ \\
\hline
\end{tabular}




\begin{tabular}{|c|c|c|c|c|}
\hline & NYISO & CAISO & SCE & Hydro-Québec \\
\hline $\begin{array}{l}\text { Wind Project } \\
\text { Data } \\
\text { Requirements }\end{array}$ & $\begin{array}{l}\text { Meteorological data } \\
\text { from at least one } \\
\text { point at least every } \\
15 \text { minutes (to be } \\
\text { increased in } 2010 \\
\text { requiring data every } \\
30 \text { seconds, and from } \\
\text { locations such that } \\
\text { no individual turbine } \\
\text { is more than } 5 \mathrm{~km} \\
\text { from a reporting } \\
\text { sensor). On-site } \\
\text { meteorological data } \\
\text { [wind speed, wind } \\
\text { direction, pressure, } \\
\text { temperature, } \\
\text { humidity, dew point] } \\
\text { from multiple } \\
\text { heights. } \\
\text { Daily penalties of the } \\
\text { greater of } \$ 500 \text { or } \\
\$ 20 / \text { MW for } \\
\text { persistent lack of or } \\
\text { bad data. }\end{array}$ & $\begin{array}{l}\text { Minimum } 1 \text { met } \\
\text { tower. } \\
\text { Proposed data } \\
\text { requirements: } \\
\text { Outage reporting: } \\
\text { Each wind project } \\
\text { must submit outages } \\
\text { of at least } 1 \mathrm{MW} \text { and } \\
\text { greater if their } \\
\text { overall capacity is } \\
\text { greater than } 10 \mathrm{MW} \text {. } \\
\text { A second met tower } \\
\text { must be installed. } \\
\text { Alternatively, a } \\
\text { second data } \\
\text { recording device may } \\
\text { be on the same met } \\
\text { tower, located at } \\
\text { approximately } 30 \\
\text { meters below the } \\
\text { average hub height. }\end{array}$ & $\begin{array}{l}\text { Meteorological } \\
\text { data (wind speed, } \\
\text { direction, } \\
\text { temperature, } \\
\text { humidity). } \\
\text { SCE uses } 12 \text { met } \\
\text { towers (six each in } \\
\text { Tehachapi and San } \\
\text { Gorgonio). SCE } \\
\text { inputs outages and } \\
\text { curtailments into } \\
\text { the system both } \\
\text { before wind } \\
\text { production (for } \\
\text { forecast } \\
\text { correction) and } \\
\text { after wind } \\
\text { production (for } \\
\text { calibration of the } \\
\text { system). }\end{array}$ & $\begin{array}{l}\text { Specifications (wind } \\
\text { project layout). } \\
\text { Real-time data } \\
\text { including: kW of active } \\
\text { power; kW power } \\
\text { available from wind } \\
\text { turbines, substation, } \\
\text { and wind project; } \\
\text { number of available } \\
\text { turbines; numbers of } \\
\text { turbines stopped due } \\
\text { to weak wind, strong } \\
\text { wind, and low } \\
\text { temperature. } \\
\text { Meteorological data } \\
\text { (horizontal and } \\
\text { vertical wind speed, } \\
\text { wind direction, } \\
\text { temperature, } \\
\text { humidity, and } \\
\text { atmospheric } \\
\text { pressure). }\end{array}$ \\
\hline
\end{tabular}




\begin{tabular}{|c|c|c|c|c|}
\hline & NYISO & CAISO & SCE & Hydro-Québec \\
\hline $\begin{array}{l}\text { Description of } \\
\text { Forecast }\end{array}$ & $\begin{array}{l}\text { Day-ahead forecasts } \\
\text { updated twice daily, } \\
\text { covering next two } \\
\text { operating days at } \\
4 \text { AM and } 4 \text { PM. } \\
\text { Real-time forecasts } \\
\text { updated every } \\
15 \text { minutes on a } \\
15 \text {-minute interval } \\
\text { basis, covering an } \\
8 \text { hour time horizon. } \\
\text { Real-time forecast is } \\
\text { blended with } \\
\text { persistence forecast } \\
\text { to develop wind } \\
\text { plant schedules in } \\
\text { real-time } \\
\text { commitment (which } \\
\text { looks ahead in } \\
15 \text {-minute intervals } \\
\text { for } 2.5 \text { hours) and } \\
\text { real-time dispatch, } \\
\text { which looks ahead in } \\
5 \text { to } 15 \text { minute } \\
\text { intervals for } 60 \\
\text { minutes. } 100 \% \\
\text { persistence used in } \\
\text { very short-term. }\end{array}$ & $\begin{array}{l}\text { Extended forecasts: } \\
\text { production (MW) for } \\
\text { each hour of days 2, } \\
\text { 3, and } 4 \text { after } \\
\text { delivery day; } \\
\text { delivered by 5:30 AM } \\
\text { on Thursdays, Fridays } \\
\text { and selected days } \\
\text { before scheduling } \\
\text { holidays. } \\
\text { Next day: production } \\
\text { (MW) for each hour } \\
\text { of next calendar day, } \\
\text { delivered by } 5: 30 \text { am. } \\
\text { Next hour: } \\
\text { production (MW) for } \\
\text { each of the next } 7 \\
\text { hours, delivered by } \\
15 \text { minutes after } \\
\text { each hour and at } \\
\text { least one hour and } \\
\text { forty-five minutes } \\
\text { before real time. }\end{array}$ & $\begin{array}{l}\text { Forecast updated } \\
\text { twice a day (at } \\
5 \mathrm{AM} \text { and } 5 \mathrm{pm}) \text {, } \\
\text { and both forecasts } \\
\text { look forward } \\
7 \text { days. }\end{array}$ & $\begin{array}{l}\text { NWPs updated twice } \\
\text { daily [at midnight and } \\
12 \text { PM (UTC)], } \\
\text { covering } 48 \text { hours } \\
\text { ahead. Additional } \\
6 \text { AM and } 6 \text { PM runs } \\
\text { operational by the end } \\
\text { of October } 2009 \text {. } \\
\text { Wind power forecasts } \\
\text { updated hourly. } \\
\text { Alarms sent to the } \\
\text { network and wind } \\
\text { project operators } \\
\text { when expected } \\
\text { storms, icy conditions, } \\
\text { or very low } \\
\text { temperatures risking } \\
\text { turbine shutdowns are } \\
\text { foreseen. }\end{array}$ \\
\hline Ramp Forecast & $\begin{array}{l}\text { No ramp forecast; } \\
\text { under consideration. }\end{array}$ & $\begin{array}{l}\text { No ramp forecast } \\
\text { currently working } \\
\text { with the DOE and } \\
\text { BPA to develop a } \\
\text { Short Term Event } \\
\text { Predictor along with } \\
\text { a ramp forecast tool. }\end{array}$ & $\begin{array}{l}\text { No ramp forecast; } \\
\text { under } \\
\text { consideration. }\end{array}$ & No ramp forecast. \\
\hline $\begin{array}{l}\text { Forecast } \\
\text { Performance } \\
\text { Metrics }\end{array}$ & $\begin{array}{l}\text { For all of the NYISO } \\
\text { and averaged for all } \\
\text { intervals between } \\
\text { June } 2008 \text { and March } \\
2009 \text { : } \\
\text { MAE of } 4.8 \% \text { for one } \\
\text { hour ahead forecast. } \\
\text { MAE of } 11.5 \% \text { for } \\
\text { day-ahead operation. }\end{array}$ & $\begin{array}{l}\text { Average RMSE for } \\
\text { the period between } \\
\text { July } 2008 \text { and July } \\
2009 \text { : } \\
\text { Day-ahead forecast: } \\
<15 \% \text { RMSE } \\
\text { Hour ahead forecast: } \\
<7 \% \text { RMSE }\end{array}$ & $\begin{array}{l}\text { Comparable wind } \\
\text { power forecast } \\
\text { performance data } \\
\text { is unavailable. }\end{array}$ & $\begin{array}{l}\text { MAE for March } 15 \text {, } \\
2009 \text { - June } 1,2009 \text { : } \\
1 \text { hr ahead: } 8.5 \% \\
4 \text { hrs ahead: } 13 \% \\
8 \text { hrs ahead: } 14.2 \% \\
12 \text { hrs ahead: } 14.4 \% \\
24 \text { hrs ahead: } 15 \% \\
\\
\text { RMSE for March } 15 \text {, } \\
2009 \text { - June } 1,2009 \text { : } \\
1 \text { hr ahead: } 12.6 \% \\
4 \text { hrs ahead: } 18 \% \\
8 \text { hrs ahead: } 19.8 \% \\
12 \text { hrs ahead: } 20.1 \% \\
24 \text { hrs ahead: } 20.7 \%\end{array}$ \\
\hline
\end{tabular}




\section{Central Wind Power Forecasting under Development in the United States and Canada}

\begin{tabular}{|c|c|c|c|c|}
\hline & $\begin{array}{l}\text { Xcel Energy } \\
\text { (Colorado) }\end{array}$ & Ontario IESO & Alberta ESO & BPA \\
\hline $\begin{array}{l}\text { Record Peak } \\
\text { Demand }\end{array}$ & $\begin{array}{l}6,884 \\
\text { (Summer 2005) }\end{array}$ & $\begin{array}{l}27,005 \mathrm{MW} \\
\text { (August 1, 2006) }\end{array}$ & $\begin{array}{l}\text { 9,806 MW } \\
\text { (December 2008) }\end{array}$ & $10,500 \mathrm{MW}$ \\
\hline $\begin{array}{l}\text { Available } \\
\text { Generating } \\
\text { Capacity }\end{array}$ & 7,738 MW & About $35,465 \mathrm{MW}$ & About $12,700 \mathrm{MW}$ & $21,580 \mathrm{MW}$ \\
\hline $\begin{array}{l}\text { Installed Wind } \\
\text { Capacity }\end{array}$ & $1,234 \mathrm{MW}$ & About $1,200 \mathrm{MW}$ & About $560 \mathrm{MW}$ & $2,284 \mathrm{MW}$ \\
\hline Area Served & $\begin{array}{l}\text { Xcel Energy serves } \\
\text { parts of eight states. } \\
\text { This information is } \\
\text { applicable only for } \\
\text { Xcel Energy's } \\
\text { operations in } \\
\text { Colorado. }\end{array}$ & Ontario, Canada & Alberta, Canada & $\begin{array}{l}300,000 \text { square miles } \\
\text { All of Washington, } \\
\text { Oregon, and Idaho, } \\
\text { and western Montana, } \\
\text { and small contiguous } \\
\text { portions of California, } \\
\text { Nevada, Utah, } \\
\text { Wyoming, and eastern } \\
\text { Montana }\end{array}$ \\
\hline $\begin{array}{l}\text { Status of } \\
\text { Centralized Wind } \\
\text { Power } \\
\text { Forecasting as of } \\
\text { November } 2009\end{array}$ & $\begin{array}{l}\text { Not yet launched. } \\
\text { Expected operation } \\
\text { date around August } \\
2010 ; \text { receipt of test } \\
\text { forecasts from NCAR } \\
\text { began September 25, } \\
2009 .\end{array}$ & $\begin{array}{l}\text { Launch of centralized } \\
\text { wind power } \\
\text { forecasting expected } \\
\text { to start in } 2010 \text {. } \\
\text { Request for proposal } \\
\text { (RFP) expected to be } \\
\text { issued in late } 2009 \text { or } \\
\text { early } 2010 \text {. }\end{array}$ & $\begin{array}{l}\text { Completed a wind } \\
\text { power forecasting } \\
\text { pilot project in } \\
2008 \text {; released } \\
\text { wind power } \\
\text { forecasting RFP } \\
\text { issued in June } \\
2009 \text {. Central wind } \\
\text { power forecasting } \\
\text { expected to start in } \\
\text { 2010. Proposes to } \\
\text { recover costs from } \\
\text { wind generators } \\
\text { via a \$/MWh } \\
\text { charge. }\end{array}$ & $\begin{array}{l}\text { Not yet launched. } \\
\text { Through 2009, } \\
\text { conducting research } \\
\text { on wind power } \\
\text { forecasting with } \\
\text { different vendors. }\end{array}$ \\
\hline $\begin{array}{l}\text { Wind Power } \\
\text { Forecast Vendor }\end{array}$ & $\begin{array}{l}\text { The National Center } \\
\text { for Atmospheric } \\
\text { Research (NCAR), } \\
\text { with assistance from } \\
\text { NREL. }\end{array}$ & $\begin{array}{l}\text { To be determined } \\
\text { through competitive } \\
\text { RFP process. }\end{array}$ & $\begin{array}{l}\text { For purposes of } \\
\text { the pilot, } 3 \text { vendors } \\
\text { were chosen: AWS } \\
\text { Truewind, Energy } \\
\text { \& Meteo Systems, } \\
\text { and WEPROG. } \\
\text { AESO expects to } \\
\text { complete } \\
\text { contracting with a } \\
\text { wind power } \\
\text { forecasting } \\
\text { provider by the } \\
\text { end of } 2009 \text {. }\end{array}$ & $\begin{array}{l}\text { To be determined by } \\
\text { January } 2010 \text { for } \\
\text { operation by end of } \\
\text { May } 2010 \text {. May opt to } \\
\text { produce the wind } \\
\text { forecast internally or } \\
\text { contract with a wind } \\
\text { forecasting company. }\end{array}$ \\
\hline
\end{tabular}




\begin{tabular}{|c|c|c|c|c|}
\hline & $\begin{array}{l}\text { Xcel Energy } \\
\text { (Colorado) }\end{array}$ & Ontario IESO & Alberta ESO & BPA \\
\hline $\begin{array}{l}\text { Forecast } \\
\text { Tools/Techniques }\end{array}$ & $\begin{array}{l}\text { NCAR will } \\
\text { incorporate } \\
\text { observations of } \\
\text { current atmospheric } \\
\text { conditions from a } \\
\text { variety of sources } \\
\text { using the Weather } \\
\text { Research and } \\
\text { Forecasting model } \\
\text { and their Real-Time } \\
\text { Four-Dimensional } \\
\text { Data Assimilation } \\
\text { system. }\end{array}$ & $\begin{array}{l}\text { Currently operating a } \\
\text { decentralized } \\
\text { forecasting regime, } \\
\text { whereby wind } \\
\text { generators submit a } \\
\text { forecast of } \\
\text { generation output. } \\
\text { Wind power forecast } \\
\text { accuracy subject to } \\
\text { compliance } \\
\text { requirements. Wind } \\
\text { generators are } \\
\text { required to provide } \\
\text { updates if actual } \\
\text { output is reasonably } \\
\text { expected to differ } \\
\text { from their original } \\
\text { forecasts by } 2 \% \text { or } 10 \\
\text { MW, whichever is } \\
\text { greater. } \\
\text { Real-time scheduling } \\
\text { done on a 5-minute } \\
\text { basis, relying on a } \\
\text { telemetry snapshot } \\
\text { of wind output from } \\
10 \text { minutes prior to } \\
\text { setting the schedule } \\
\text { in real time. } \\
\text { Decentralized } \\
\text { forecasting regime } \\
\text { will eventually be } \\
\text { replaced by } \\
\text { centralized wind } \\
\text { power forecasting } \\
\text { (expected to be in } \\
\text { service in } 2010 \text { ). }\end{array}$ & $\begin{array}{l}\text { In the pilot project: } \\
\text { - AWS Truewind } \\
\text { utilized the eWind } \\
\text { system, which } \\
\text { produced forecasts } \\
\text { via an assembly of } \\
\text { physics-based } \\
\text { (NWP) and } \\
\text { statistical models. } \\
\text { - Energy \& Meteo } \\
\text { used NWP input, a } \\
\text { combination of } \\
\text { several numerical } \\
\text { weather models } \\
\text { weighted } \\
\text { according to the } \\
\text { weather situation, } \\
\text { site-specific power } \\
\text { curves based on } \\
\text { historical data, and } \\
\text { shortest-term } \\
\text { model (0-10 hours) } \\
\text { based on power } \\
\text { measurements. } \\
\text { - WEPROG used an } \\
\text { ensemble } \\
\text { forecasting system } \\
\text { with a large } \\
\text { number of } \\
\text { members, based } \\
\text { on their Wind } \\
\text { Generation Pool } \\
\text { (WGP) and NWP } \\
\text { forecasts. }\end{array}$ & $\begin{array}{l}\text { Forecasts to be made } \\
\text { up to } 36 \text { hours ahead } \\
\text { at four wind projects; } \\
\text { two in Oregon and } \\
\text { two in Washington. } \\
\text { Will focus particularly } \\
\text { on wind ramps. BPA } \\
\text { has also installed } 14 \\
\text { met devices in their } \\
\text { Balancing Authority } \\
\text { Area. Data feeds from } \\
\text { met sites will be in } \\
\text { place by late } 2009 \text { for } \\
\text { forecast displays. } \\
\text { BPA will be developing } \\
\text { a "wind desk" over the } \\
\text { next two years to help } \\
\text { dispatchers. }\end{array}$ \\
\hline
\end{tabular}




\begin{tabular}{|c|c|c|c|c|}
\hline & Xcel Energy & Ontario IESO & Alberta ESO & BPA \\
\hline $\begin{array}{l}\text { Data } \\
\text { Requirements }\end{array}$ & $\begin{array}{l}\text { Collect turbine-level } \\
\text { and onsite met tower } \\
\text { detail from wind } \\
\text { projects into the Xcel } \\
\text { energy scheduling } \\
\text { system. They are } \\
\text { also pursuing remote } \\
\text { sensing technologies } \\
\text { for short-term } \\
\text { forecasting purposes. }\end{array}$ & To be determined. & $\begin{array}{l}\text { For the pilot, } \\
\text { 10-minute facility } \\
\text { power (MW) } \\
\text { output: historical } \\
\text { and real time; } \\
\text { 10-minute wind } \\
\text { speed, wind } \\
\text { direction, } \\
\text { temperature and } \\
\text { pressure: historical } \\
\text { and real time; } \\
\text { 10-minute real- } \\
\text { time turbine } \\
\text { availability (at } 2 \\
\text { sites only). } \\
\text { Subject to AESO } \\
\text { rulemaking, AESO } \\
\text { will impose data } \\
\text { requirements on } \\
\text { wind plants, i.e., } \\
\text { 10-minute met } \\
\text { tower and wind } \\
\text { generation, and } \\
\text { available capacity. } \\
\text { Operational power } \\
\text { limit will be used } \\
\text { as well. } \\
\text { Forecasts to } \\
\text { incorporate up to } \\
\text { two years of } \\
\text { historical met and } \\
\text { power data. }\end{array}$ & $\begin{array}{l}\text { Wind power } \\
\text { forecasters to use } \\
\text { metrological data } \\
\text { taken from wind } \\
\text { facilities. }\end{array}$ \\
\hline
\end{tabular}




\begin{tabular}{|c|c|c|c|c|}
\hline & Xcel Energy & Ontario IESO & Alberta ESO & BPA \\
\hline $\begin{array}{l}\text { Forecast Update } \\
\text { Frequency }\end{array}$ & $\begin{array}{l}\text { 3-km nested } \\
\text { forecasting grid } \\
\text { updated every } \\
3 \text { hours. }\end{array}$ & $\begin{array}{l}\text { As per existing } \\
\text { market rules, the } \\
\text { market participants } \\
\text { submit day-ahead } \\
\text { wind power forecast } \\
\text { by } 11: 00 \text { AM on a } \\
\text { daily basis. } \\
\text { Additional forecast } \\
\text { updates are required } \\
\text { as conditions change. } \\
\text { Some wind } \\
\text { companies update } \\
\text { their forecast on } \\
\text { hourly basis while } \\
\text { others may not } \\
\text { frequently update } \\
\text { apart from the } \\
\text { day-ahead forecast. } \\
\text { Market Assessment } \\
\text { and Compliance } \\
\text { Division (MACD) of } \\
\text { the IESO looks for } \\
\text { evidence that the } \\
\text { market participant } \\
\text { has exercised due } \\
\text { diligence in } \\
\text { revising/updating } \\
\text { dispatch data } \\
\text { (forecasts) to reflect } \\
\text { changed conditions } \\
\text { or expected } \\
\text { injection. } \\
\text { Existing } \\
\text { processes/rules will } \\
\text { be updated upon } \\
\text { start of centralized } \\
\text { wind power } \\
\text { forecasting (expected } \\
\text { to be in service in } \\
\text { 2010). }\end{array}$ & $\begin{array}{l}\text { For the pilot, three } \\
\text { forecasters } \\
\text { delivered a } \\
\text { 48-hour forecast, } \\
\text { updated each } \\
\text { hour. Forecasts } \\
\text { were delivered in } \\
\text { real time for } \\
7 \text { existing wind } \\
\text { generation } \\
\text { facilities (WGFs), } \\
5 \text { future WGFs as } \\
\text { well as } 4 \text { regional } \\
\text { aggregates of } 3 \\
\text { WGFs each, and } \\
\text { the aggregate of } \\
\text { the existing WGFs, } \\
\text { future WGFs and } \\
\text { all WGFs. } \\
\text { For the RFP, AESO } \\
\text { wants hourly } \\
\text { forecasts up to } \\
48 \text { hours ahead; } \\
\text { and max and min } \\
\text { forecast at several } \\
\text { confidence levels; } \\
\text { and forecasted } \\
\text { maximum ramp } \\
\text { rates within hour } \\
\text { and notification of } \\
\text { multi-hour large } \\
\text { ramps. AESO } \\
\text { interested in } \\
\text { expanding } \\
\text { forecasting to } 72, \\
96, \text { or } 120 \text { hours } \\
\text { ahead and rolling } \\
6 \text {-hour intra-hour } \\
\text { forecast at } \\
\text { 10-minute } \\
\text { intervals. }\end{array}$ & $\begin{array}{l}\text { Forecast likely } \\
\text { updated every ten } \\
\text { minutes. It will have } \\
10 \text {-minute predictions } \\
\text { out } 6 \text { hours, and } \\
\text { hourly predictions out } \\
36 \text { hours. }\end{array}$ \\
\hline
\end{tabular}




\section{Table References}

\section{PJM}

Focken, Ulrich and Lange, Matthias, 2007, Summary of Forecast Methodology of Previento, prepared by Energy \& Meteo GmbH, June 25, 2007, http://www.aeso.ca/downloads/EMSYS_Generic_Summary.pdf.

Personal Communication, Sanjay Patil, PJM Interconnection, August 21, 2009.

Personal Communication, Ulrich Focken, Energy \& Meteo Systems, August 20, 2009.

PJM, 2009, PJM 2008 Annual Report, issued April 2009, http://www.pjm.com/about-pjm/who-weare/ /media/about-pjm/newsroom/2008-annual-report.ashx.

PJM, 2009, “PJM Training Course: Wind Generation Operational Considerations”, June 9, 2009, slides.

PJM Intermittent Resource Working Group, 2009, meeting minutes PJM Interconnection, January 8, 2009, http://www.pjm.com/Media/committees-groups/working-groups/irwg/20090108-minutes.pdf.

PJM Power System Coordination Department, 2009, PJM Manual 14D: Generator Operational Requirements, Revision 15; effective April 1, 2009, http://www.pjm.com/documents/ /media/documents/manuals/m14d.ashx.

\section{ERCOT}

Botterud, Audun and Wang, Jianhui, 2009, Wind Power Forecasting and Electricity Market Operations, http://www.usaee.org/usaee2009/submissions/OnlineProceedings/Botterud_etal_paper.pdf.

Doggett, Trip, 2009, "Renewables Development", ERCOT presentation for the Gulf Coast Power Association Spring Conference, April 3, 2009, slides, http://www.gulfcoastpower.org/default/s09doggett.pdf.

ERCOT, 2008a, EMS Wind Power Forecasting Detailed Design (B2) v1.0, March 4, 2008.

ERCOT, 2008b, PRR794: Meteorological Data Required From QSEs Representing Wind-Powered Generation Resources, effective date: 04/01/2009 http://www.ercot.com/mktrules/issues/prr/775-799/794/.

ERCOT, 2009, “ERCOT Quick Facts”, May 2009, http://www.ercot.com/content/news/presentations/ 2009/ERCOT\%20Quick\%20Facts\%20May\%202009.pdf.

Personal Communication, David Maggio, Electric Reliability Council of Texas, Inc, September 2, 2009.

Personal Communication, Kenneth Pennock, AWS Truewind, LLC, September 4, 2009. 
Saathoff, Kent, 2009, "Grid Operations and Planning Report", presentation to the ERCOT Board of Directors, November 17, 2009, slides, http://www.ercot.com/content/meetings/board/keydocs/ 2009/1117/Item_08_-_Grid_Operations_and_Planning_Report.pdf.

Zack, John W, 2008, "Wind Forecasting Efforts to Improve Renewable Penetration", AWS Truewind presentation for the Integrated Energy Policy Report workshop, Sacramento, CA, July 31, 2008, slides, http://www.energy.ca.gov/2008_energypolicy/documents/2008-07-31_workshop/presentations/ Wind_Forecasting_Efforts-Zack.pdf.

Zack, John W, 2009, "Overview of the Current Status and Future Prospects of Wind Power Production Forecasting for the ERCOT System", AWS Truewind presentation at an ERCOT workshop, Austin, TX, June 26, 2009, slides.

\section{Midwest ISO}

Botterud, Audun and Wang, Jianhui, 2009, Wind Power Forecasting and Electricity Market Operations, http://www.usaee.org/usaee2009/submissions/OnlineProceedings/Botterud_etal_paper.pdf.

Focken, Ulrich and Lange, Matthias, 2007, Summary of Forecast Methodology of Previento, prepared by Energy \& Meteo GmbH, June 25, 2007, http://www.aeso.ca/downloads/EMSYS_Generic_Summary.pdf.

Midwest ISO, 2009, “Corporate Information", November 2009, http://www.midwestiso.org/publish/Document/ 3e2d0_106c60936d4_-7ba50a48324a/Corporate\%20Fact\%20Sheet\%209-1-09.pdf?action= download\&_property=Attachment.

Midwest ISO, 2009, Guide to Day-ahead Intermittent Resource Forecast Data Submittal for Reliability, March 31, 2009, http://www.midwestmarket.org/publish/Document/2c41ee_1200f54a695_-7dd80a48324a/ Intermittent\%20Resource\%20Forecast\%20Submittal\%20Guide\%20(2).pdf?action=download\&_property= Attachment.

Ontario IESO, 2009, Centralized Renewable Forecasting, draft, February 4, 2009, http://www.ieso.ca/imoweb/pubs/consult/se57/se57-20090210-Wind-Forecasting.pdf.

Personal Communication, Joe Sullivan, WindLogics, August 26, 2009.

Personal Communication, Kris Ruud, Midwest ISO, November 19, 2009.

Personal Communication, Michael McMullen, Midwest ISO, August 28, 2009.

Personal Communication, Ulrich Focken, Energy \& Meteo Systems, August 20, 2009.

\section{NYISO}

AWS Truewind, Forecasting: eWind ${ }^{\circledR}$ - Proven, Accurate, Valuable, http://www.awstruewind.com/forecasting.cfm/details/true (accessed: June 2009). 
Botterud, Audun and Wang, Jianhui, 2009, Wind Power Forecasting and Electricity Market Operations, http://www.usaee.org/usaee2009/submissions/OnlineProceedings/Botterud_etal_paper.pdf.

Edelson, David, 2009a, "Changing Operating Standards for Wind in New York: Forecasting and Curtailments", AWEA wind and transmission workshop: grid operations and integration session, Overland Park, KS, March 17-18, 2009, slides.

Edelson, David, 2009b, "Requirements and Expectations for a Quality Wind Forecast", NYISO presentation for the wind energy production forecasting webinar, February 9, 2009, slides, http://www.uwig.org/webinar20909/Edelson.pdf.

Edelson, David, 2009c, "Wind Forecasting and Curtailment Experience at NYISO", NYISO presentation for the UWIG Fall Technical Workshop, Cedar Rapids, lowa, October 7-9, 2009, slides, http://www.uwig.org/iowawork/Edelson.pdf.

Federal Energy Regulatory Commission, Order Conditionally Accepting Tariff Revisions, 123 FERC $₫$ 61,267, June 17, 2008.

Gonzales, Rick, 2009, "Operational Challenges and Innovative Solutions to Integrating Renewable Resources", NYISO presentation, Washington, DC, March 2, 2009, slides, http://www.ferc.gov/EventCalendar/Files/20090303120300-Gonzales,\%20NYISO.pdf.

NYISO, 2009, 2009 Load and Capacity Data: "Gold Book", version 2, released August 2009.

NYISO Market Issues Working Group, 2007, “Wind Rules and Forecasting Project Update”, draft, December 14, 2007, slides, http://www.nyiso.com/public/webdocs/committees/bic_miwg/meeting_materials/2007-1214/MIWG_Wind_Forecasting_and_Rules_20071214_v2.pdf.

Ontario IESO, 2009b, Improving Forecasts From Variable Generation, June 11, 2009, http://www.ieso.com/imoweb/pubs/consult/se57/se57-20090616-Centralized-Wind-Forecasting.pdf.

Personal Communication, David Edelson, New York ISO, August 17, 2009.

Personal Communication, Erik Ela, National Renewable Energy Laboratory, July 13, 2009.

Pike, Robert, 2008, “Wind Rules and Forecasting Proposal”, January 16, 2008, NYISO slides, http://www.nyiso.com/public/webdocs/committees/bic/meeting_materials/2008-0116/BIC_agenda_07_Wind_Forecasting_and_Rules_Proposal_11608.pdf.

\section{CAISO}

AWS Truewind, Forecasting: eWind ${ }^{\circledR}$ - Proven, Accurate, Valuable, http://www.awstruewind.com/forecasting.cfm/details/true (accessed: June 2009). 
Blatchford, Jim, 2008, "CAISO Supporting Integration of Renewables", BPA/CAISO international wind forecasting workshop, July 24-25, 2008, slides, http://www.bpa.gov/corporate/business/innovation/docs/2008/ BPA_California\%20ISO\%20Cal\%20ISO\%20Presentation.pdf.

Botterud, Audun and Wang, Jianhui, 2009, Wind Power Forecasting and Electricity Market Operations, http://www.usaee.org/usaee2009/submissions/OnlineProceedings/Botterud_etal_paper.pdf.

California Energy Commission. Summer 2009 Electricity Supply and Demand Outlook. CEC-200-2009-007, May 2009. http://www.energy.ca.gov/2009publications/CEC-200-2009-007/CEC-200-2009-007.PDF.

CAISO, 2009, 2008 California ISO Annual Report, posted September 9, 2009, http://www.caiso.com/2424/2424b1b6278c0.pdf.

CAISO, 2009, Settlements \& Billing: BPM Configuration Guide: Forecasting Service Fee, CC 701, version 1.10, March 12, 2009, http://www.caiso.com/1b6a/1b6aa70416258.doc.

Helman, Udi, 2009, "Examination of Operational or Dispatch Provisions of Wholesale Tariffs and Market Rules", FERC technical conference: integrating renewable resources into the wholesale electric grid, remarks by Udi Helman, March 2, 2009, http://www.ferc.gov/eventcalendar/Files/20090302091501Helman,\%20CAISO.pdf.

Ontario IESO, 2009, Improving Forecasts From Variable Generation, June 11, 2009, http://www.ieso.com/imoweb/pubs/consult/se57/se57-20090616-Centralized-Wind-Forecasting.pdf.

Personal Communication, Jim Blatchford, California ISO, August 14, 2009.

Zack, John W, 2005, "Wind Power Production Forecasting for CAISO PIRP", workshop on wind resource forecasting and RTO/ISO integration, hosted by PJM Interconnection, March 3, 2005, slides.

Zack, John W, 2008, "Wind Forecasting Efforts to Improve Renewable Penetration", AWS Truewind presentation for the integrated energy policy report workshop, Sacramento, CA, July 31, 2008, slides, http://www.energy.ca.gov/2008_energypolicy/documents/2008-07-31_workshop/ presentations/Wind_Forecasting_Efforts-Zack.pdf.

\section{$\underline{\text { SCE }}$}

AWS Truewind, Forecasting: eWind ${ }^{\circledR}$ - Proven, Accurate, Valuable, http://www.awstruewind.com/forecasting.cfm/details/true (accessed: June 2009).

Bailey, Bruce; Brower, Michael; Cheng, Min; Gilman, Barry; Isaac, Jerry; and Zack, John, 2001, The Value of Wind Forecasting to Southern California Edison, http://d.wanfangdata.com.cn/NSTLHY_NSTL_HY2163883.aspx.

Personal Communication, Arthur Canning, Southern California Edison, November 20, 2009.

Personal Communication, Barry Gilman, Southern California Edison, June 26, 2009. 


\section{$\underline{\text { Xcel Energy }}$}

Parks, Keith, 2009, “Xcel Wind Forecasting Project”, UWIG spring forecasting workshop, February 18-19, 2009 slides.

Personal Communication, Craig Cox, Interwest, November 30, 2009.

Personal Communication, Erik Ela, National Renewable Energy Laboratory, July 13, 2009.

Personal Communication, Keith Parks, Xcel Energy, August 17, 2009.

Personal Communication, Mark Ahlstrom, WindLogics, September 2, 2009.

Public Service Company of Colorado, Notice of Filing of Reduced Firm Demand and Energy Sales Forecasts, before the Public Utilities Commission of the State of Colorado, Docket No. 07A-447E, March 19, 2009, http://www.xcelenergy.com/SiteCollectionDocuments/ docs/07A447E_PSCoNoticeFilingReducedDemandEnergySalesForecasts_03-19-09.pdf.

University Corporation for Atmospheric Research, 2009, "NCAR Forecasts Will Help Xcel Energy Harness Wind", news release, February 4, 2009, http://www.ucar.edu/news/releases/2009/xcel.jsp.

\section{Ontario IESO}

Ontario IESO, Demand Overview, http://www.ieso.ca/imoweb/media/md_demand.asp (accessed November 19, 2009)

Ontario IESO, Supply Overview, http://www.ieso.ca/imoweb/media/md_supply.asp (accessed November 19, 2009).

Ontario IESO, 2008, "Near Term Wind Forecasting: Improving Final Pre-Dispatch Results Through the Utilization of Persistence Forecasting", presentation to the Wind Power Standing Committee, May 14, 2008, slides, http://www.ieso.ca/imoweb/pubs/consult/windpower/wpsc-20080514-Item4.pdf.

Ontario IESO, 2009a, Centralized Renewable Forecasting, draft, February 4, 2009, http://www.ieso.ca/imoweb/pubs/consult/se57/se57-20090210-Wind-Forecasting.pdf.

Ontario IESO, 2009b, "Information and Discussion: Centralized Forecasting for Variable Generation", June 16, 2009, slides, http://www.ieso.ca/imoweb/pubs/consult/se57/se57-20090616-Centralized-Wind-Forecastingpres.pdf.

Ontario IESO, 2009c, "Ontario's System Operator to Launch Centralized Wind Forecasting", news release, August 18, 2009, http://www.ieso.ca/imoweb/media/md_newsitem.asp?newsID=4842.

Personal Communication, Martin Hastings, Ontario IESO, August 18, 2009. 


\section{Hydro-Québec}

Balvet, Vincent; Bourret, Jacques; and Maubois, Jean-Emmanuel, 2009, Massive Data Acquisition for the Short Term Wind Power Forecasting in a Large Grid: The Hydro-Québec SAGIPE System Experience, Power Systems Conference and Exposition, 2009, PSCE apos;09, IEEE/PES, 15-18 March 2009, Page(s):1 -7, digital object identifier: 10.1109/PSCE.2009.4839924.

Forcione, Alain; Petrucci, Franco; Roberge, Gaétan; and Yu, Wei, 2008, "Hydro-Québec and Environment Canada Wind Energy Forecasting Project", NE Region System Operators' wind integration seminar, Montréal, February 13, 2008, slides, http://www.weican.ca/documents/2008/080213-19-AlainForcioneForecasting\%20NE\%20Region\%20Wind\%20Integration.pdf.

Hydro-Québec, 2009, Powering Our Future: Annual Report 2008, http://www.hydroquebec.com/publications/en/annual_report/index.html.

Hydro-Québec Distribution, 2009, “Electricity Supply for Québec Needs: Call for Tenders Document A/O 2009-02, Addendum 1", issue date: June 8, 2009, APPENDIX VII.

Personal Communication, Alain Forcione, Hydro-Québec, August 31, 2009.

Personal Communication, Francis Gosselin, Hydro-Québec, November 20, 2009.

Personal Communication, Jacques Bourret, Hydro-Québec, August 31, 2009.

\section{Alberta ESO}

Alberta Electric System Operator, Current Supply Demand Report, http://ets.aeso.ca/ets_web/ip/Market/Reports/CSDReportServlet (accessed November 19, 2009).

Alberta Electric System Operator, Wind Power Forecasting Pilot Project, http://www.aeso.ca/gridoperations/13825.html (accessed June 2009).

Alberta Electric System Operator, 2009a, Powering Albertans, volume 3, issue 1, posted March 5, 2009, http://www.aeso.ca/downloads/Powering_Albertans_FINAL.pdf.

Alberta Electric System Operator, 2009b, "Wind Integration Stakeholder Session”, August 12, 2009, slides, http://www.aeso.ca/downloads/AESO_Stakeholder_Session_Presentation_(Aug_12)_(2).pdf.

Alberta Electric System Operator, 2009c, Wind Power Forecasting Service: Request for Proposals, date issued: June 23, 2009, http://www.aeso.ca/downloads/Wind_Power_Forecast_RFP_FINAL_23June09.pdf.

AWS Truewind, LLC, 2008, AWS Truewind's Final Report for the Alberta Forecasting Pilot Project, submitted: June 25, 2008, http://www.aeso.ca/downloads/Alberta_PP_Final_Report_AWST_Jun25.pdf. 
Focken, Ulrich and Lange, Matthias, 2008, Final report: Wind power forecasting pilot project in Alberta, Canada, Energy \& Meteo Systems GmbH, May 26, 2008, http://www.aeso.ca/downloads/Final_report_emsys_lv.pdf.

Frost, Warren, 2009, Final recommendations regarding implementation of market and operational framework (MOF) for wind integration in Alberta and AESO response to stakeholder comments, June 18, 2009, http://www.aeso.ca/downloads/MOF_stakeholder_comments_and_AESO_response_matrix_cover_letter.pdf.

Industry Work Group, 2008, Wind Power Forecasting Pilot Project: Industry Work Group Report, Alberta Department of Energy, Alberta Electric System Operator, Alberta Energy Research Institute, August 6, 2008, http://www.aeso.ca/downloads/Work_Group_Paper_Final_(3).pdf.

Jørgensen, Jess and Möhrlen, Corinna, 2008, AESO Wind Power Forecasting Pilot Project: Final Project Report, WEPROG, May 2008, http://www.aeso.ca/downloads/weprog_pilotproject_finalreport_v4.pdf.

Ontario IESO, 2009a, Centralized Renewable Forecasting, draft, February 4, 2009, http://www.ieso.ca/imoweb/pubs/consult/se57/se57-20090210-Wind-Forecasting.pdf.

Personal Communication, James Shen, Alberta ESO, September 1, 2009.

Personal Communication, Mark Ahlstrom, WindLogics, September 2, 2009.

\section{BPA}

Bonneville Power Administration, 2009, "2008 BPA Facts”, http://www.bpa.gov/corporate/about_BPA/Facts/FactDocs/BPA_Facts_2008.pdf.

Bonneville Power Authority, 2009, "Wind Generation Capacity in the BPA Balancing Authority Area", September 22, 2009, http://www.transmission.bpa.gov/business/operations/Wind/WIND_InstalledCapacity_current.xls.

Bonneville Power Administration, 2009, “Wind Integration Initiatives Update”, September 18, 2009, slides.

Personal Communication, Bart McManus, Bonneville Power Administration, August 17, 2009.

Personal Communication, Doug Larson, Western Interstate Energy Board, December 2, 2009.

Personal Communication, Doug Johnson, Bonneville Power Administration, December 3, 2009.

Renewable Energy World, 2009, “BPA Sponsors Wind Forecast Competition”, August 6, 2009, http://www.renewableenergyworld.com/rea/news/article/2009/08/bpa-sponsors-wind-forecastcompetition. 


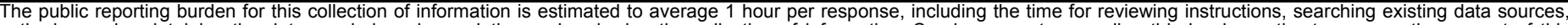

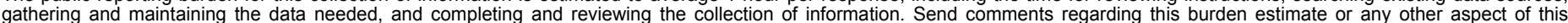

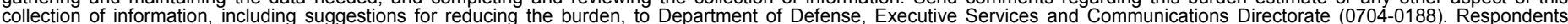

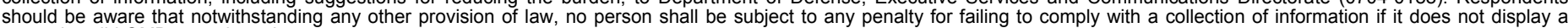

should be aware that notwithstanding

PLEASE DO NOT RETURN YOUR FORM TO THE ABOVE ORGANIZATION.

\begin{tabular}{l|l|l|l} 
1. REPORT DATE $(D D-M M-Y Y Y Y)$ & 2. & REPORT TYPE & 3. DATES COVERED (FrOm - TO)
\end{tabular}

December 2009

4. TITLE AND SUBTITLE

Central Wind Power Forecasting Programs in North America by

Regional Transmission Organizations and Electric Utilities

5a. CONTRACT NUMBER

DE-AC36-08-GO28308

5b. GRANT NUMBER

5c. PROGRAM ELEMENT NUMBER

6. AUTHOR(S)

K. Porter and J. Rogers

5d. PROJECT NUMBER

NREL/SR-550-46763

5e. TASK NUMBER

WER9.5501

5f. WORK UNIT NUMBER
7. PERFORMING ORGANIZATION NAME(S) AND ADDRESS(ES)

Exeter Associates, 5565 Sterrett Place, Suite 310, Columbia, MD 21044
8. PERFORMING ORGANIZATION REPORT NUMBER

LAM-9-99431-01

9. SPONSORING/MONITORING AGENCY NAME(S) AND ADDRESS(ES)

National Renewable Energy Laboratory

10. SPONSOR/MONITOR'S ACRONYM(S)

NREL

1617 Cole Blvd.

Golden, CO 80401-3393

11. SPONSORING/MONITORING AGENCY REPORT NUMBER NREL/SR-550-46763

12. DISTRIBUTION AVAILABILITY STATEMENT

National Technical Information Service

U.S. Department of Commerce

5285 Port Royal Road

Springfield, VA 22161

13. SUPPLEMENTARY NOTES

NREL Technical Monitor: Jeff Hein

14. ABSTRACT (Maximum 200 Words)

The report addresses the implementation of central wind power forecasting by electric utilities and regional transmission organizations in North America.

\section{SUBJECT TERMS}

Wind; integration; forecasting; regional transmission organizations; RTOs; utilties; North America; electrical

16. SECURITY CLASSIFICATION OF:
\begin{tabular}{|l|l|l|}
\hline a. REPORT & b. ABSTRACT & c. THIS PAGE \\
Unclassified & Unclassified & Unclassified \\
& & \\
\hline
\end{tabular}

\begin{tabular}{|c|c|}
\hline $\begin{array}{l}\text { 17. LIMITATION } \\
\text { OF ABSTRACT }\end{array}$ & $\begin{array}{l}\text { 18. } \\
\text { NUMBER } \\
\text { OF PAGES }\end{array}$ \\
\hline UL & \\
\hline
\end{tabular}

19a. NAME OF RESPONSIBLE PERSON

19b. TELEPHONE NUMBER (Include area code) 